\title{
Entry of severe fever with thrombocytopenia syndrome virus
}

\author{
Fei Yuan ${ }^{1 凶}$, Aihua Zheng ${ }^{2 \bowtie}$ \\ 1. CAS Key Laboratory of Pathogenic Microbiology and Immunology, Institute of Microbiology, Chinese \\ Academy of Sciences, Beijing 100101, China \\ 2. State Key Laboratory of Integrated Management of Pest Insects and Rodents, Institute of Zoology, \\ Chinese Academy of Sciences, Beijing 100101, China
}

Severe fever with thrombocytopenia syndrome virus (SFTSV) is a globe-shaped virus covered by a dense icosahedral array of glycoproteins $\mathrm{Gn} / \mathrm{Gc}$ that mediate the attachment of the virus to host cells and the fusion of viral and cellular membranes. Several membrane factors are involved in virus entry, including $C$-type lectins and nonmuscle myosin heavy chain IIA. The post-fusion crystal structure of the Gc protein suggests that it is a class II membrane fusion protein, similar to the E/E1 protein of flaviviruses and alphaviruses. The virus particles are internalized into host cell endosomes through the clathrin-dependent pathway, where the low pH activates the fusion of the virus with the cell membrane. With information from studies on other bunyaviruses, herein we will review our knowledge of the entry process of SFTSV.

\section{KEYWORDS severe fever with thrombocytopenia syndrome virus (SFTSV); bunyavirus; entry; fusion; receptor}

\section{INTRODUCTION}

The Bunyaviridae family of viruses includes five genera-Orthobunyavirus, Hantavirus, Nairovirus, Phlebovirus, and Tospovirus-with over 350 different viruses isolated to date. With the exception of hantaviruses, all bunyaviruses are transmitted by arthropods infecting diverse vertebrates, invertebrates, and plants (Tospovirus is the only genus that infects plants). Many bunyaviruses are important human pathogens, causing severe diseases including encephalitis, hepatitis, and hemorrhagic fever. Severe fever with thrombocytopenia syndrome virus (SFTSV) is an emerging tick-borne pathogen that was first described in China in 2009 (Yu et al., 2011) and later was also isolated in Japan (Takahashi et al., 2014) and Korea (Denic et al., 2011). A similar virus, named

Received: 28 August 2016, Accepted: 30 November 2016,

Published online: 16 December 2016

$\triangle$ Correspondence:

Fei Yuan, Phone: +86-10-64807569, Fax: +86-10-64807598,

Email: yuanf@im.ac.cn

ORCID: 0000-0003-3053-7063

Aihua Zheng, Phone: +86-10-64807178, Fax: +86-10-64807099,

Email: zhengaihua@ioz.ac.cn

ORCID: 0000-0002-2184-227X the Heartland virus, was reported in 2012 from two patients in Missouri, USA (Mcmullan et al., 2012). The clinical symptoms of SFTSV infections include fever, thrombocytopenia, gastrointestinal disorder, and leukocytopenia, with a fatality rate of $2 \%-30 \%$ based on different reports (Yu et al., 2011; Liu Q et al., 2014; Liu S et al., 2014). SFTSV is a novel phlebovirus that encapsidates three segments of negative-sense or ambisense RNA, named small (S), medium (M), and large (L) (Figure 1A). The S RNA encodes the nucleocapsid protein $\mathrm{N}$ and non-structural protein NS, the M RNA encodes the glycoproteins $\mathrm{Gn} / \mathrm{Gc}$, and the L RNA encodes the viral RNA-dependent RNA polymerase (Figure 1B). The glycoproteins $\mathrm{Gn} / \mathrm{Gc}$ are incorporated into the envelope of the virus particles to orchestrate both the docking of the virus particles to host cells and the low $\mathrm{pH}$-dependent fusion of the virus and cell membranes during endocytic entry (Figure 2). In this review, we will discuss recent progress towards understanding the entry mechanism of SFTSV.

\section{VIRUS STRUCTURE}

Bunyaviruses are enveloped, high-ordered spherical vi- 
A

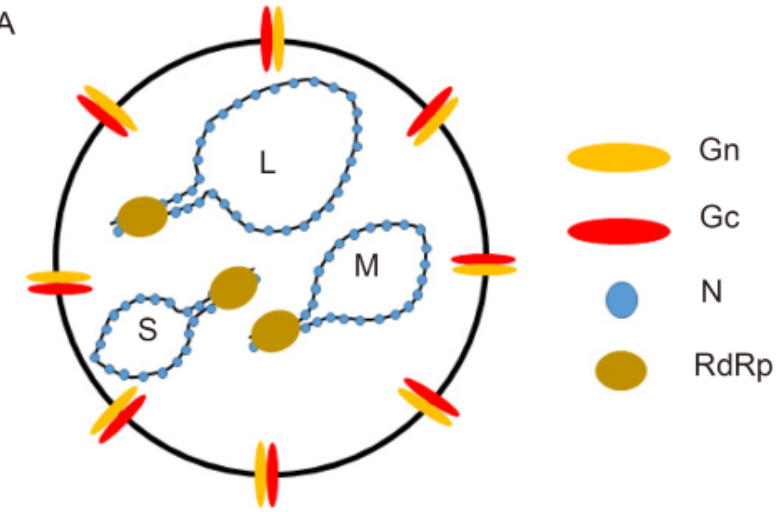

B
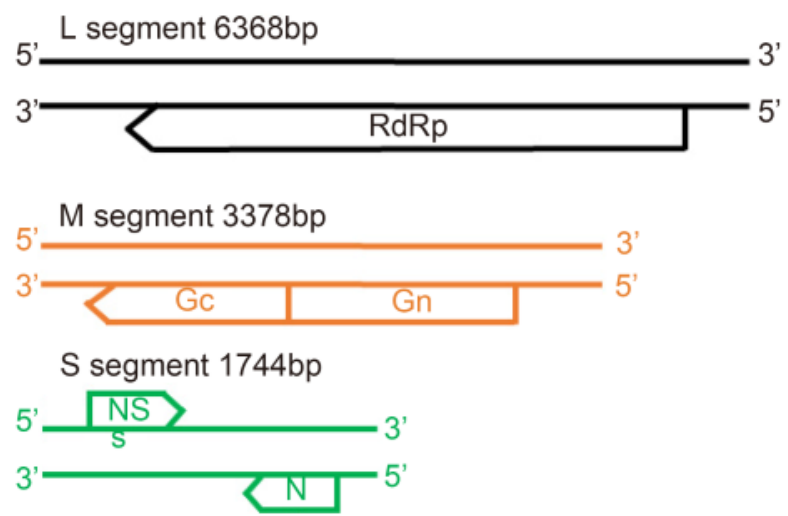

Figure 1. Schematic representation of the SFTSV virion and genome organization. (A) SFTSV particles are enveloped and spherical shaped, with the Gn-Gc dimer incorporated on the surface. The SFTSV genome consists of three segments, namely S/M/L, which are based on the size. (B) Arrows below each segment indicate the ORFs transcribed from the negative-sense templates, while the arrow above the segment shows the ORF transcribed from the positive-sense template.

ruses with a diameter of $90-110 \mathrm{~nm}$. The viral envelope is composed of a lipid bilayer and two transmembrane glycoproteins, $\mathrm{Gn} / \mathrm{Gc}$, regularly arranged on the surface. Although the SFTSV virion structure has barely been studied, electron cryotomography of the related phlebovirus Uukuniemi (UUKV) virus suggests that the spikes formed by $\mathrm{Gn} / \mathrm{Gc}$ arrange in an icosahedral lattice, with $\mathrm{T}=12$ triangulation (Overby et al., 2008). Two distinct spike organization forms have been discovered and are found to be $\mathrm{pH}$-dependent, which is similar to the low $\mathrm{pH}$-triggered conformational changes observed in alphaviruses and flaviviruses (Overby et al., 2008; Yu et al., 2008). The ribonucleoproteins (RNPs) formed by the RNA genome and nucleoprotein appear as a thread that partially interacts with the viral envelope (Overby et al.,
2008). Similar results have also been reported for Rift Valley Fever Virus (RVFV), another phlebovirus. At a 2.1-2.3 nm resolution, the capsomers resemble hollow cylinders incorporated at five- and six-coordinated positions. The RNPs are also visible and strongly interact with the cytoplasmic tail of the glycoproteins, most likely Gn (Huiskonen et al., 2009).

\section{THE ENVELOPE PROTEIN}

The SFTSV glycoproteins mediate virus entry by binding to cellular receptors and inducing the fusion of the virus and the cell membrane during endocytosis. They are also the sole targets for neutralization antibodies. The $M$ segment of the SFTSV encodes the Gn and Gc glycoproteins. In addition, the $\mathrm{M}$ segments of some bunyaviruses have also been found to encode an NSm protein; however, sequence analysis suggests that SFTSV does not have such a protein (Marklewitz et al., 2011; Yu et al., 2011). Like in other bunyaviruses, in SFTSV as well, $\mathrm{Gn}$ and $\mathrm{Gc}$ are first synthesized as a $\mathrm{Gn} / \mathrm{Gc}$ precursor polyprotein in the secretory compartment of the infected cells, and the process then continues by proteolytic cleavage (Antic et al., 1992). The $\mathrm{Gn} / \mathrm{Gc}$ precursor protein is cleaved by a signal peptidase during trafficking into the endoplasmic reticulum (ER) (Lober et al., 2001; Gerrard and Nichol, 2007) and then modified by Nlinked glycosylation (Kuismanen, 1984). Upon trafficking to the Golgi apparatus, the glycan is further processed into hybrid and complex forms (Madoff and Lenard, 1982; Shi et al., 2005). When expressing Gn or Gc alone in the cell, Gn is transported to the Golgi apparatus owing to its Golgi localization motif, whereas Gc is localized to the ER owing to its ER retention signal (Gerrard and Nichol, 2002). However, when co-expressing $\mathrm{Gn}$ and $\mathrm{Gc}$ with a vaccinia virus vector, both $\mathrm{Gn}$ and Gc localize to the Golgi apparatus, which suggests that there a physical interaction occurs between Gn and Gc after cleavage (Wasmoen et al., 1988). Bunyavirus particles usually bud into the lumen of the Golgi apparatus during virus assembly, which is facilitated by Gn and Gc (Spiropoulou, 2001; Novoa et al., 2005; Overby et al., 2006; Piper et al., 2011; Cifuentes-Munoz et al., 2014).

\section{FUSION OF THE VIRUS AND THE HOST CELL MEMBRANE}

The bunyavirus envelope glycoprotein Gc has been postulated to be a class II fusion protein based on proteomic and computational analyses (Garry CE and Garry RT, 2004; Rusu et al., 2012). Consistent with this hypothesis, Gc of hantaviruses is found to interact with artificial membranes (Tischler et al., 2005). Dr. Yorgo Modis's 


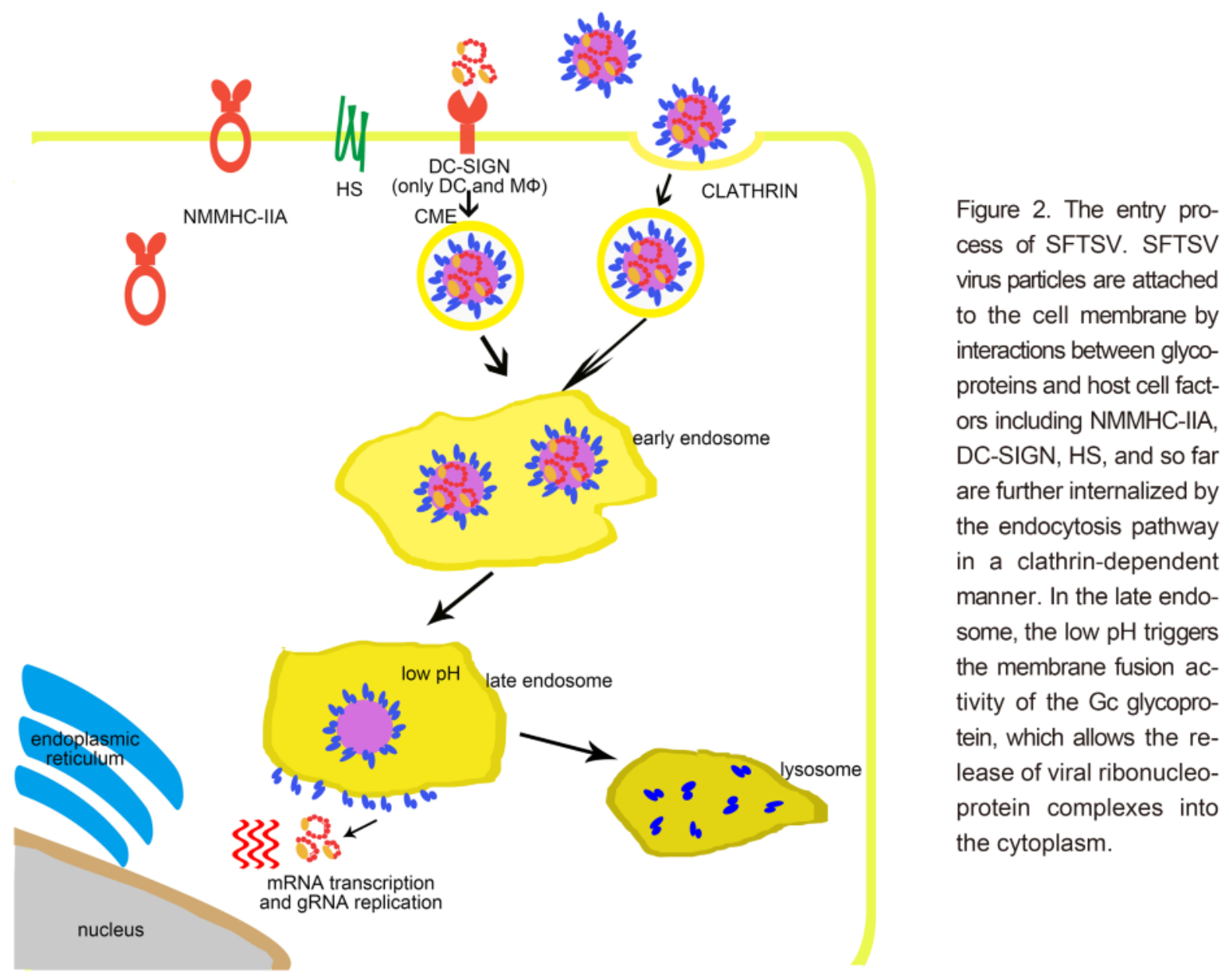

laboratory has solved the crystal structure of the RVFV Gc protein, which shows a pre-fusion conformation of the class II fusion protein previously found in alphaviruses and flaviviruses (Dessau and Modis, 2013). The extracellular domain of the RVFV Gc protein folds into a three-domain structure. Domain I is a 10-stranded barrel located in the middle, the mostly stranded domain II is extended from domain I, with the fusion loop on its tip, and domain III is an IgC-like module connected to domain I via a flexible linker (Dessau and Modis, 2013). The recently solved post-fusion structure of SFTSV Gc reveals the conformational changes triggered by low $\mathrm{pH}$ during virus entry (Halldorsson et al., 2016). Similar to the E1 protein of alphaviruses, the recombinant SFTSV $\mathrm{Gc}$ ectodomain is produced as a monomer at $\mathrm{pH} 8.0$ (Pierson and Kielian, 2013). However, at pH 5.0, a trimer peak appears on size exclusion without interacting with artificial lipid membrane, as for most class II fusion proteins (Dessau and Modis, 2013). The crystal structure produced from this peak at low $\mathrm{pH}$ reveals a clear class II post-fusion conformation.

Comparison of the pre-fusion structure of RVFV Gc and post-fusion structure of SFTSV Gc reveals that there is a major conformational change occurring in the modules during fusion. Although the pre- and post-fusion conformations of DIII are very similar, major structural rearrangements are observed upon overlay of domains I and II (Dessau and Modis, 2013). Domain I refolds into a secondary structure, which also occurs in the fusogenic rearrangement of the flavivirus (Modis et al., 2004) and alphavirus fusion proteins (Gibbons et al., 2004). Domain I and II of three monomers are packed into a trimer core, while domain III shifts $24 \AA$ toward the core, thus forming the outer layer of the Gc trimer (Dessau and Modis, 2013).

The mechanism underlying class II membrane fusion has been intensively studied for alphaviruses and flaviviruses (Pierson and Kielian, 2013). The fusion process involves a series of conformational changes in the fusion protein triggered by the mildly acidic endosomal environment and includes several quick steps. Exposure of virus particles to low $\mathrm{pH}$ dissociates the E1/E2 (alphavirus) or the $\mathrm{E}_{2}$ (flavivirus) dimer and rotates the domain II from the viral membrane. The fusion loop on the tip of domain II is thus exposed and inserted into the host membrane. Domain I/II of E or E1 forms a trimer core 
via protein-protein interactions. DIII then rotates towards the trimer core and forms the outer layer of the $\mathrm{E}$ or E1 trimer. DIII also triggers the movement of the transmembrane domain, together with the viral membrane, towards the host membrane, thus resulting in the fusion of the virus and the host cell membranes (Kielian and Rey, 2006; Kielian et al., 2010).

\section{ATTACHMENT FACTORS AND VIRUS RECEPTOR}

Cellular receptor or receptors are the key host factors involved in the virus entry process. The interaction between viral glycoproteins and host receptors plays essential roles in determining cellular tropism, endocytosis, and/or activating the fusion process. To make the following discussion more clear, we defined virus receptors as the membrane components that precisely and specifically bind to viral glycoproteins and that are essential for viral entry. On the other hand, attachment factors were defined as membrane components that can enhance viral entry efficiency by interacting with viral particles but are ultimately dispensable for entry. Multiple factors such as dendritic cell-specific intercellular adhesion molecule-3grabbing non-integrin (DC-SIGN), heparan sulfate (HS), and Nonmuscle Myosin Heavy Chain IIA (NMMHCIIA) have been identified to be involved in the entry of SFTSV and other phleboviruses.

\section{(1) DC-SIGN}

During natural transmission, bunyaviruses are injected into the skin during blood feeding by virus-carrying arthropods. At the initial infection, dermal dendritic cells (DCs) are among the first target cells. DC-SIGN is specifically expressed on the surface of dermal DCs and is a calciumdependent C-type lectin specialized for the capture and presentation of foreign antigens (van Kooyk, 2008). DCSIGN is a type II transmembrane protein that binds with high-mannose N-glycans (Svajger et al., 2010), which are typical for insect-derived arbovirus glycoproteins. Therefore, alphaviruses and flaviviruses have been shown to infect immature DCs depending on DC-SIGN (Navarro-Sanchez et al., 2003; Tassaneetrithep et al., 2003; Svajger et al., 2010). There are also many reports suggesting that DC-SIGN facilitates the entry of many phleboviruses including UUKV, RVFV, Punta Toro virus (PTV), Toscana virus (TOSV) (Lozach et al., 2011), La Crosse virus (LACV), and SFTSV (Hofmann et al., 2013). UUKV and RVFV infect DCs and DC-SIGN-expressing cell lines through the binding of DC-SIGN and high-mannose N-glycans on $\mathrm{Gn} / \mathrm{Gc}$ proteins (Lozach et al., 2011). Upon virus inoculation, virus-induced clustering on the cell surface can be visualized by live cell imaging (Lozach et al., 2011). An endocytosis-defective mutant DC-SIGN does not mediate virus infection, which indicates that DC-SIGN is both an attachment factor and an authentic receptor. Upon docking on the cell membrane by binding to DC-SIGN, phleboviruses are delivered into cells due to the endocytotic signals in the cytoplasmic tail of DC-SIGN (Engering et al., 2002). In the early endosome, the virus particles dissociate from DC-SIGN and continue into the late endosome where membrane fusion happens (Lozach et al., 2011). A recent study has revealed that DC-SIGN, DC-SIGN-related (DC-SIGNR), and liver and lymph node sinusoidal endothelial cell C-type lectin (LSECtin) function as SFTSV receptors (Tani et al., 2016). However, there are many phlebovirus-susceptible cell lines that do not express lectins (Lozach et al., 2011), which indicates that other unidentified receptors for phlebovirus entry must exist. The presence of such receptors is also strongly suggested by the broad cell tropism of phleboviruses and the very limited tissue expression of DC-SIGN and other lectins.

\section{(2) HS}

All eukaryotic cells are covered by a dense and diverse layer of carbohydrates, which is essential for many biological processes (Varki, 2007). It is not a surprise that many viruses have evolved to exploit these ubiquitous and accessible surface glycans to promote their entry process (Liu and Thorp, 2002). Among these different types of glycans, HS serves as an attachment factor for many viruses (Liu and Thorp, 2002) including RVFV (de Boer et al., 2012). HS is a glycosaminoglycan (GAG), i.e., a linear polysaccharide that can be attached to membrane proteins to form proteoglycans. HS is abundantly expressed on most cell lines (Liu and Thorp, 2002). Using $\mathrm{CHO}$ cell lines with defined glycosylation defects, HS was revealed to be required for the efficient entry of RVFV. RVFV entry was also found to be significantly reduced by pre-incubating the virus with sulfated heparin or pre-treating the cells with heparinase (de Boer et al., 2012). Although RVFV infection on HS-defective cell lines was dramatically reduced, residual infection was still observed, which suggested that HS is more likely an attachment factor rather than a receptor (de Boer et al., 2012). The involvement of HS in RVFV entry has also been confirmed by a haploid screening conducted for RVFV spreading factor (Heijink et al., 2015). More evidence suggests that CCHFV and Hantaan virus (HNTV) also rely on HS for efficient entry, while Andes virus (ANDV) does not (Heijink et al., 2015). However, the involvement of HS in SFTSV entry is still under investigation.

\section{(3) NMMHC-IIA}

NMMHC-IIA is an actin-binding motor protein that is 
normally involved in cell migration, adhesion, polarization, and morphogenesis (Vicente-Manzanares et al., 2009). NMMHC-IIA has also been shown to function as a herpes simplex virus type 1 (HSV-1) receptor, and NMMHC-IIA expression levels on the cell surface are increased upon HSV-1 infection (Arii et al., 2010). In 2014, Sun et al. reported that the extracellular domain of recombinant Gn protein binds to SFTSV-susceptible cell lines and blocks infection. Using Gn as bait, the authors identified NMMHC-IIA as an SFTSV entry factor by using a co-IP strategy (Sun et al., 2014). The authors also found that overexpression of NMMHC-IIA enhanced SFTSV infection in HeLa cells. Moreover, RNAi knockdown of NMMHC-IIA and the use of an anti-NMMHCIIA antibody were found to reduce SFTSV infection but did not completely block it (Sun et al., 2014). These data suggested that NMMHC-IIA is an attachment factor. As NMMHC-IIA is highly expressed on platelets and is essential for their normal functions, it is plausible that NMMHC-IIA directly contributes to the pathogenesis of SFTSV (Sun et al., 2014). An in vitro study of primary cells suggested that SFTSV virus particles can efficiently infect macrophages but can only bind to platelets. Another study revealed that SFTSV adheres to platelets and promotes the phagocytosis of SFTSV-platelet complexes by macrophages, thus resulting in thrombocytopenia (Jin et al., 2012). Collectively, these results indicate that NMMHC-IIA might play important roles in SFTSV entry and pathogenesis; however, the bona fide receptor for SFTSV entry is still unknown.

\section{ENTRY PATHWAY}

The fusion of SFTSV with the host cell membrane requires pH lower than 6 (Hofmann et al., 2013; Tani et al., 2016). A prerequisite for the activation of the SFTSV fusion protein $\mathrm{Gc}$ is the uptake of virions into the host cell endosomes (Tani et al., 2016). In a previous study, inhibitor analysis revealed that SFTSV entry could be blocked by dynasore, a dynamin inhibitor, which indicated that SFTSV entry depends on clathrin (Hofmann et al., 2013). Orthobunyavirus and Oropouche viruses also enter host cells via clathrin-coated pits (Santos et al., 2008; Hollidge et al., 2012). However, a different detailed study of the entry of UUKV suggested that UUKV internalization is mainly clathrin independent (Lozach et al., 2010). The authors reported that the virus enters Rab5a+ and LAMP-1+ late endosomes, where acidactivated penetration happens (Lozach et al., 2010). Together, these data suggest that different bunyaviruses use different internalization mechanisms for their entry, and the detailed processes involved in also remain to be investigated.

\section{CONCLUSION}

Substantial progress has been made in understanding the entry mechanism of SFTSV after its discovery as an emerging virus in 2009. However, the discovery of the SFTSV Gc protein as a class II fusion protein will especially provide important insights into the membrane fusion mechanism of the whole Bunyaviridae family. However, the critical receptor that determines cell tropism and entry is still unknown, although many attachment factors have been identified. Therefore, more efforts should be devoted to SFTSV research in the future.

\section{ACKNOWLEDGMENTS}

This work was funded by the National Key Plan for Scientific Research and Development of China (2016YFD0500300), the Strategic Priority Research Program of the Chinese Academy of Sciences (CAS) (XDB11030800), and the Natural Science Foundation of China (L1524009).

\section{COMPLIANCE WITH ETHICS GUIDELINES}

The authors declare that they have no conflict of interest. This article does not contain any studies with human or animal subjects performed by any of the authors.

\section{REFERENCES}

Antic D, Wright KE, Kang CY. 1992. Maturation of Hantaan virus glycoproteins G1 and G2. Virology, 189: 324-328.

Arii J, Goto H, Suenaga T, Oyama M, Kozuka-Hata H, Imai T, Minowa A, Akashi H, Arase H, Kawaoka Y, Kawaguchi Y. 2010. Non-muscle myosin IIA is a functional entry receptor for herpes simplex virus-1. Nature, 467: 859-862.

Cifuentes-Munoz N, Salazar-Quiroz N, Tischler ND. 2014. Hantavirus $\mathrm{Gn}$ and $\mathrm{Gc}$ envelope glycoproteins: key structural units for virus cell entry and virus assembly. Viruses, 6: 1801-1822.

de Boer SM, Kortekaas J, de Haan CA, Rottier PJ, Moormann RJ, Bosch BJ. 2012. Heparan sulfate facilitates Rift Valley fever virus entry into the cell. J Virol, 86: 13767-13771.

Denic S, Janbeih J, Nair S, Conca W, Tariq WU, Al-Salam S. 2011. Acute Thrombocytopenia, Leucopenia, and Multiorgan Dysfunction: The First Case of SFTS Bunyavirus outside China? Case Rep Infect Dis, 2011: 204056.

Dessau M, Modis Y. 2013. Crystal structure of glycoprotein C from Rift Valley fever virus. Proc Natl Acad Sci U S A, 110: 1696-1701.

Engering A, Geijtenbeek TB, van Vliet SJ, Wijers M, van Liempt E, Demaurex N, Lanzavecchia A, Fransen J, Figdor CG, Piguet V, van Kooyk Y. 2002. The dendritic cell-specific adhesion receptor DC-SIGN internalizes antigen for presentation to $\mathrm{T}$ cells. J Immunol, 168: 2118-2126.

Garry CE, Garry RF. 2004. Proteomics computational analyses suggest that the carboxyl terminal glycoproteins of Bunyaviruses are class II viral fusion protein (beta-penetrenes). Theor Biol Med Model, 1: 10. 
Gerrard SR, Nichol ST. 2002. Characterization of the Golgi retention motif of Rift Valley fever virus $\mathrm{G}(\mathrm{N})$ glycoprotein. J Virol, 76: $12200-12210$.

Gerrard SR, Nichol ST. 2007. Synthesis, proteolytic processing and complex formation of N-terminally nested precursor proteins of the Rift Valley fever virus glycoproteins. Virology, 357: 124-133.

Gibbons DL, Vaney M-C, Roussel A, Vigouroux A, Reilly B, Lepault J, Kielian M, Rey FA. 2004. Conformational change and protein-protein interactions of the fusion protein of Semliki Forest virus. Nature, 427: 320-325.

Halldorsson S, Behrens AJ, Harlos K, Huiskonen JT, Elliott RM, Crispin M, Brennan B, Bowden TA. 2016. Structure of a phleboviral envelope glycoprotein reveals a consolidated model of membrane fusion. Proc Natl Acad Sci U S A, 113: 7154-7159.

Heijink AM, Blomen VA, Bisteau X, Degener F, Matsushita FY, Kaldis P, Foijer F, van Vugt MA. 2015. A haploid genetic screen identifies the G1/S regulatory machinery as a determinant of Wee1 inhibitor sensitivity. Proc Natl Acad Sci U S A, 112: $15160-15165$.

Hofmann H, Li X, Zhang X, Liu W, Kuhl A, Kaup F, Soldan SS, Gonzalez-Scarano F, Weber F, He Y, Pohlmann S. 2013. Severe fever with thrombocytopenia virus glycoproteins are targeted by neutralizing antibodies and can use DC-SIGN as a receptor for $\mathrm{pH}$-dependent entry into human and animal cell lines. J Virol, 87: 4384-4394.

Hollidge BS, Nedelsky NB, Salzano MV, Fraser JW, GonzalezScarano F, Soldan SS. 2012. Orthobunyavirus entry into neurons and other mammalian cells occurs via clathrin-mediated endocytosis and requires trafficking into early endosomes. $\mathrm{J}$ Virol, 86: 7988-8001.

Huiskonen JT, Overby AK, Weber F, Grunewald K. 2009. Electron cryo-microscopy and single-particle averaging of Rift Valley fever virus: evidence for GN-GC glycoprotein heterodimers. J Virol, 83: 3762-3769.

Jin C, Liang M, Ning J, Gu W, Jiang H, Wu W, Zhang F, Li C, Zhang Q, Zhu H, Chen T, Han Y, Zhang W, Zhang S, Wang Q, Sun L, Liu Q, Li J, Wang T, Wei Q, Wang S, Deng Y, Qin C, Li D. 2012. Pathogenesis of emerging severe fever with thrombocytopenia syndrome virus in C57/BL6 mouse model. Proc Natl Acad Sci U S A, 109: 10053-10058.

Kielian M, Chanel-Vos C, Liao M. 2010. Alphavirus entry and membrane fusion. Viruses, 2: 796-825.

Kielian M, Rey FA. 2006. Virus membrane fusion proteins: more than one way to make a hairpin. Nat Rev Microbiol, 4: 67-76.

Kuismanen E. 1984. Posttranslational processing of Uukuniemi virus glycoproteins G1 and G2. J Virol, 51: 806-812.

Liu J, Thorp SC. 2002. Cell surface heparan sulfate and its roles in assisting viral infections. Med Res Rev, 22: 1-25.

Liu Q, He B, Huang SY, Wei F, Zhu XQ. 2014. Severe fever with thrombocytopenia syndrome, an emerging tick-borne zoonosis. Lancet Infect Dis, 14: 763-772.

Liu S, Chai C, Wang C, Amer S, Lv H, He H, Sun J, Lin J. 2014. Systematic review of severe fever with thrombocytopenia syndrome: virology, epidemiology, and clinical characteristics. Rev Med Virol, 24: 90-102.

Lober C, Anheier B, Lindow S, Klenk HD, Feldmann H. 2001. The Hantaan virus glycoprotein precursor is cleaved at the conserved pentapeptide WAASA. Virology, 289: 224-229.

Lozach PY, Kuhbacher A, Meier R, Mancini R, Bitto D, Bouloy M, Helenius A. 2011. DC-SIGN as a receptor for phleboviruses. Cell Host Microbe, 10: 75-88.

Lozach PY, Mancini R, Bitto D, Meier R, Oestereich L, Overby AK, Pettersson RF, Helenius A. 2010. Entry of bunyaviruses in- to mammalian cells. Cell Host Microbe, 7: 488-499.

Madoff DH, Lenard J. 1982. A membrane glycoprotein that accumulates intracellularly: cellular processing of the large glycoprotein of LaCrosse virus. Cell, 28: 821-829.

Marklewitz M, Handrick S, Grasse W, Kurth A, Lukashev A, Drosten C, Ellerbrok H, Leendertz FH, Pauli G, Junglen S. 2011. Gouleako virus isolated from West African mosquitoes constitutes a proposed novel genus in the family Bunyaviridae. J Virol, 85: 9227-9234.

McMullan LK, Folk SM, Kelly AJ, MacNeil A, Goldsmith CS, Metcalfe MG, Batten BC, Albarino CG, Zaki SR, Rollin PE, Nicholson WL, Nichol ST. 2012. A new phlebovirus associated with severe febrile illness in Missouri. N Engl J Med, 367 : 834-841.

Modis Y, Ogata S, Clements D, Harrison SC. 2004. Structure of the dengue virus envelope protein after membrane fusion. Nature, 427: 313-319.

Navarro-Sanchez E, Altmeyer R, Amara A, Schwartz O, Fieschi F, Virelizier JL, Arenzana-Seisdedos F, Despres P. 2003. Dendriticcell-specific ICAM3-grabbing non-integrin is essential for the productive infection of human dendritic cells by mosquito-cellderived dengue viruses. EMBO Rep, 4: 723-728.

Novoa RR, Calderita G, Cabezas P, Elliott RM, Risco C. 2005. Key Golgi factors for structural and functional maturation of bunyamwera virus. J Virol, 79: 10852-10863.

Overby AK, Pettersson RF, Grunewald K, Huiskonen JT. 2008. Insights into bunyavirus architecture from electron cryotomography of Uukuniemi virus. Proc Natl Acad Sci U S A, 105: 2375-2379.

Overby AK, Popov V, Neve EP, Pettersson RF. 2006. Generation and analysis of infectious virus-like particles of uukuniemi virus (bunyaviridae): a useful system for studying bunyaviral packaging and budding. J Virol, 80: 10428-10435.

Pierson TC, Kielian M. 2013. Flaviviruses: braking the entering. Curr Opin Virol, 3: 3-12.

Piper ME, Sorenson DR, Gerrard SR. 2011. Efficient cellular release of Rift Valley fever virus requires genomic RNA. PLoS One, 6: e18070.

Rusu M, Bonneau R, Holbrook MR, Watowich SJ, Birmanns S, Wriggers W, Freiberg AN. 2012. An assembly model of rift valley Fever virus. Front Microbiol, 3: 254.

Santos RI, Rodrigues AH, Silva ML, Mortara RA, Rossi MA, Jamur MC, Oliver C, Arruda E. 2008. Oropouche virus entry into HeLa cells involves clathrin and requires endosomal acidification. Virus Res, 138: 139-143.

Shi X, Brauburger K, Elliott RM. 2005. Role of N-linked glycans on bunyamwera virus glycoproteins in intracellular trafficking, protein folding, and virus infectivity. J Virol, 79: 13725-13734.

Spiropoulou CF. 2001. Hantavirus maturation. Curr Top Microbiol Immunol, 256: 33-46.

Sun Y, Qi Y, Liu C, Gao W, Chen P, Fu L, Peng B, Wang H, Jing Z, Zhong G, Li W. 2014. Nonmuscle myosin heavy chain IIA is a critical factor contributing to the efficiency of early infection of severe fever with thrombocytopenia syndrome virus. J Virol, 88: 237-248.

Svajger U, Anderluh M, Jeras M, Obermajer N. 2010. C-type lectin DC-SIGN: an adhesion, signalling and antigen-uptake molecule that guides dendritic cells in immunity. Cell Signal, 22: $1397-1405$.

Takahashi T, Maeda K, Suzuki T, Ishido A, Shigeoka T, Tominaga T, Kamei T, Honda M, Ninomiya D, Sakai T, Senba T, Kaneyuki S, Sakaguchi S, Satoh A, Hosokawa T, Kawabe Y, Kurihara S, Izumikawa K, Kohno S, Azuma T, Suemori K, Yasukawa M, Mizutani T, Omatsu T, Katayama Y, Miyahara M, 
Ijuin M, DoiK, Okuda M, Umeki K, Saito T, Fukushima K, Nakajima K, Yoshikawa T, Tani H, Fukushi S, Fukuma A, Ogata M, Shimojima M, Nakajima N, Nagata N, Katano H, Fukumoto H, Sato Y, Hasegawa H, Yamagishi T, Oishi K, Kurane I, Morikawa S, Saijo M. 2014. The first identification and retrospective study of Severe Fever with Thrombocytopenia Syndrome in Japan. J Infect Dis, 209: 816-827.

Tani H, Shimojima M, Fukushi S, Yoshikawa T, Fukuma A, Taniguchi S, Morikawa S, Saijo M. 2016. Characterization of Glycoprotein-Mediated Entry of Severe Fever with Thrombocytopenia Syndrome Virus. J Virol, 90: 5292-5301.

Tassaneetrithep B, Burgess TH, Granelli-Piperno A, Trumpfheller C, Finke J, Sun W, Eller MA, Pattanapanyasat K, Sarasombath S, Birx DL, Steinman RM, Schlesinger S, Marovich MA. 2003. DC-SIGN (CD209) mediates dengue virus infection of human dendritic cells. J Exp Med, 197: 823-829.

Tischler ND, Gonzalez A, Perez-Acle T, Rosemblatt M, Valenzuela PD. 2005. Hantavirus Gc glycoprotein: evidence for a class II fusion protein. JGV, 86: 2937-2947.

van Kooyk Y. 2008. C-type lectins on dendritic cells: key modulators for the induction of immune responses. Biochem Soc Trans, 36: 1478-1481.
Varki A. 2007. Glycan-based interactions involving vertebrate sialic-acid-recognizing proteins. Nature, 446: 1023-1029.

Vicente-Manzanares M, Ma X, Adelstein RS, Horwitz AR. 2009. Non-muscle myosin II takes centre stage in cell adhesion and migration. Nat Rev Mol Cell Biol, 10: 778-790.

Wasmoen TL, Kakach LT, Collett MS. 1988. Rift Valley fever virus $\mathrm{M}$ segment: cellular localization of $\mathrm{M}$ segment-encoded proteins. Virology, 166: 275-280.

Yu IM, Zhang W, Holdaway HA, Li L, Kostyuchenko VA, Chipman PR, Kuhn RJ, Rossmann MG, Chen J. 2008. Structure of the immature dengue virus at low $\mathrm{pH}$ primes proteolytic maturation. Science, 319: 1834-1837.

Yu XJ, Liang MF, Zhang SY, Liu Y, Li JD, Sun YL, Zhang L, Zhang QF, Popov VL, Li C, Qu J, Li Q, Zhang YP, Hai R, Wu W, Wang Q, Zhan FX, Wang XJ, Kan B, Wang SW, Wan KL, Jing HQ, Lu JX, Yin WW, Zhou H, Guan XH, Liu JF, Bi ZQ, Liu GH, Ren J, Wang H, Zhao Z, Song JD, He JR, Wan T, Zhang JS, Fu XP, Sun LN, Dong XP, Feng ZJ, Yang WZ, Hong T, Zhang Y, Walker DH, Wang Y, Li DX. 2011. Fever with thrombocytopenia associated with a novel bunyavirus in China. N Engl J Med, 364: 1523-1532. 